Chirurg 2017 $\cdot 88: 345$

DOI 10.1007/s00104-017-0419-y

Online publiziert: 29. März 2017

๑) Springer Medizin Verlag GmbH 2017

CrossMark

F. Rauchfuß $\cdot$ U. Settmacher

Klinik für Allgemein-, Viszeral- und Gefäßchirurgie, Universitätsklinikum Jena, Jena, Deutschland

\title{
Stellenwert des
}

Lymphknotenstatus bei Patienten mit hilären Cholangiokarzinom

deshalb aus der weiteren Analyse ausgeschlossen wurden. Es wurden in der Mehrzahl linksseitige Hemihepatektomien durchgeführt $(n=96 ; 54,9 \%)$. Bei 143 Patienten $(81,7 \%)$ konnte eine R0-Situation erreicht werden.

Bei den 175 Patienten, die schlussendlich analysiert wurden, waren insgesamt 1133 Lymphknoten entnommen worden (Median: 6,5; 1-25). Bei 70 Patienten (40\%) musste ein metastatischer Lymphknotenbefall diagnostiziert werden. Es zeigte sich eine deutliche Korrelation zwischen T-Stadium und Lymphknotenbefall: T1 - Lymphknoten bei 11,1\% der Patienten befallen, T2 - 23,5\% und T3/4 - 45,7 ( $p=0,01)$.

Die 5-Jahres-Überlebensrate betrug $32,1 \%$. In einer multivariaten Analyse waren ein R1-Status sowie das Vorliegen von Lymphknotenmetastasen mit einem schlechten Outcome assoziiert.

Bei den nodal positiven Patienten betrug die LK-Ration im Mittel 0,41. Eine Ratio >0,2 war mit einem signifikant schlechteren Überleben (10,6\% vs. $24,4 \%, p=0,04)$ assoziiert. Die absolute Anzahl befallener Lymphknoten spielte hinsichtlich des Überlebens in dieser Subgruppe keine Rolle.

In der Gruppe der nodal negativen $\mathrm{Pa}$ tienten zeigte sich ein deutlich schlechteres Outcome, wenn weniger als 6 Lymphknoten entnommen wurden $(34,2 \%$ vs. $64,5 \%)$.

Fazit. Die hier vorgestellte Studie unterstreicht den prognostischen Stellenwert des R-Status sowie des Lymphknotenstatus. Erstmals konnte an einer großen
Fallzahl zudem die Rolle der LK-Ratio herausgearbeitet werden. Ob dies beispielweise bei der Festlegung adjuvanter Therapiekonzepte Berücksichtigung finden wird, sollte Gegenstand zukünftiger Studien sein.

Es konnte zudem für das perihiläre Cholangiokarzinom gezeigt werden, dass eine Mindestanzahl von 5 entnommenen Lymphknoten eine prognostische Bedeutung hat und deshalb zu fordern ist.

\section{Korrespondenzadresse}

PD Dr. F. Rauchfuß, M.Sc.

Klinik für Allgemein-, Viszeral- und Gefäßchirurgie, Universitätsklinikum Jena Am Klinikum 1, 07747 Jena, Deutschland falk.rauchfuss@med.uni-jena.de

Interessenkonflikt. F. Rauchfuß und U. Settmacher geben an, dass kein Interessenkonflikt besteht. 